\title{
Diethyl Sulfate
}

National Cancer Institute

\section{Source}

National Cancer Institute. Diethyl Sulfate. NCI Thesaurus. Code C44375.

A colorless, corrosive, oily liquid that darkens with age and has a faint peppermint odor. Diethyl sulfate is mainly used as an ethylating agent in organic synthesis and in the dye and textile manufacturing. Exposure to this substance results in severe irritation to the eyes, skin and respiratory tract. It is a possible mutagen and is reasonably anticipated to be a human carcinogen based on evidence of carcinogenicity in experimental animals and may be associated with developing laryngeal cancer. (NCI05) 